\title{
Desenvolvimento de um modelo de pé segmentado para avaliação de indivíduos calçados
}

\author{
Development of a segmented foot model for \\ assessing individuals with shoes
}

\author{
Renan Alves Resende ${ }^{[a]}$, Lucas Rodrigues Nascimento ${ }^{[a]}$, Maria Clarice Lopes Silva ${ }^{[b]}$, \\ Ana Cisalpino Pinheiro ${ }^{[b]}$, Sérgio Teixeira Fonseca ${ }^{[c]}$, Renata Noce Kirkwood ${ }^{[d]}$
}

[a] Doutorandos em Ciências da Reabilitação da Universidade Federal de Minas Gerais (UFMG), Belo Horizonte, MG - Brasil, e-mails: renanresende@hotmail.com,lrn@ufmg.br

[b] Acadêmicas do Curso de Fisioterapia da Universidade Federal de Minas Gerais (UFMG), Belo Horizonte, MG - Brasil, e-mails: mcla.lopes@yahoo.com.br, anacisalpino@gmail.com

[c] Professor associado do Departamento de Fisioterapia da Universidade Federal de Minas Gerais (UFMG), Belo Horizonte, MG - Brasil, e-mail: sfonseca@eeffto.ufmg.br

[d] Professora adjunta do Departamento de Fisioterapia da Universidade Federal de Minas Gerais (UFMG), Belo Horizonte, MG - Brasil, e-mail: renata.kirkwood@gmail.com

\section{Resumo}

Introdução: 0 uso de calçados pode influenciar parâmetros cinemáticos dos segmentos do pé durante a marcha. Objetivo: Testar um modelo biomecânico para avaliar o deslocamento angular dos segmentos do pé durante a marcha de indivíduos calçados. Materiais e métodos: Dez indivíduos adultos jovens saudáveis participaram do estudo. Um par de tênis foi utilizado durante o estudo. 0 sistema Qualisys Pró-Reflex foi utilizado para a avaliação das variáveis de desfecho: deslocamento angular de retropé nos planos sagital, frontal e transverso e de antepé nos planos frontal e transverso. 0 Coeficiente de Correlação Intraclasse foi usado para verificar o grau de associação das seguintes variáveis entre as duas visitas: valores angulares máximos e mínimos e deslocamento angular total. Resultados: As variáveis de desfecho apresentaram confiabilidade teste-reteste de moderada a excelente. Discussão: 0 nível de confiabilidade encontrado foi considerado aceitável, fornecendo, assim, uma base sólida para a avaliação objetiva da cinemática dos segmentos do pé de indivíduos calçados em ambientes laboratoriais, para fins de pesquisas científicas e avaliações clínicas da cinemática da 
marcha humana. Conclusão: A qualidade do método desenvolvido e da análise de confiabilidade realizada detectou padrões de movimento dos segmentos do pé próximos aos descritos na literatura. Além disso, o protocolo de aquisição apresentado não é invasivo, não requer uma estrutura extra de referência, posicionamento da articulação subtalar em neutro nem é dependente da informação fornecida por raios-X.

Palavras-chave: Cinemática. Pé. Marcha. Confiabilidade. Calçados.

\section{Abstract}

Introduction: The use of shoes may influence the kinematics parameters of the foot segments during gait. objective: To test a biomechanical model developed to measure the angular displacement of the foot segments during gait of individuals using shoes. Materials and methods: Ten healthy young individuals participated in the study. A pair of shoes was used during the study. The Qualisys Pró-Reflex system was used to measure the following outcome variables: angular displacement of the rearfoot in the sagittal, frontal and transverse planes of motion and forefoot in the frontal and transverse planes. The Intraclass Correlation Coefficient was calculated to verify the association between the following variables between visits: maximum and minimum joint angles and the total range of motion. Results: The investigated outcomes demonstrated values of intra-rater reliability ranging from moderate to excellent. Discussion: This study presented a feasible and reliable method to measure the angular displacement of the foot segments during gait using shoes. The methods can be applied during scientific researches and clinical assessments of the kinematics during human gait. Conclusion: The quality of the method developed and the reliability analysis realized demonstrated motion patterns of the segments of the foot similar to what is demonstrated in the literature. In addition, the protocol is noninvasive, does not require an additional reference device, subtalar neutral position and does not depend on information provided by X-ray images.

Keywords: Kinematics. Foot. Gait. Reliability. Shoes.

\section{Introdução}

A perna e o complexo do pé são importantes componentes do sistema músculo-esquelético responsáveis pela interação entre o membro inferior e a superfície de apoio (1) permitindo, dessa forma, a adequada distribuição das cargas inerciais e gravitacionais durante a marcha humana (2). Anormalidades no pé ou tornozelo podem causar distribuição irregular das cargas impostas ao sistema musculoesquelético e determinar adaptações compensatórias que impõem demandas excessivas em diversas estruturas do membro inferior, favorecendo o surgimento de patologias insidiosas, inclusive em regiões proximais $(3,4)$.

Evidências científicas indicaram que corredores classificados como pronadores excessivos apresentam maior valgismo dinâmico de joelho durante a fase de contato inicial o que poderia levar a uma maior sobrecarga sobre o compartimento medial do joelho (5). Nesse contexto, a eversão excessiva do calcâneo durante a fase de apoio da marcha é usualmente mencionada como um dos possíveis fatores determinantes de modificações no padrão cinemático de joelho e quadril $(6,7)$. Tais achados demonstram a influência dos movimentos das articulações do complexo do pé nos demais segmentos do membro inferior e reforçam a importância de uma adequada avaliação desses movimentos durante a análise de marcha.

Embora os movimentos do pé apresentem relativa especificidade e complexidade devido à eminente característica multiplanar das superfícies de contato das articulações do pé, os modelos biomecânicos clínicos utilizados usualmente representam o pé como um vetor rígido único, o que permite que apenas os ângulos de progressão do pé de flexão plantar e de dorsiflexão sejam determinados $(8,9)$. Tais modelos são insuficientes para a caracterização dos movimentos do pé em três dimensões durante a fase de apoio da marcha (10).

Carson et al. (8) desenvolveram um modelo biomecânico de pé segmentado associado a um protocolo de avaliação aplicável à análise da marcha. Foram demonstrados padrões de movimento das diferentes partes do pé consistentes com o conhecimento existente acerca da cinemática do pé e as medidas apresentaram confiabilidade aceitável, o que somado 
a outros modelos previamente descritos $(2,8,11)$, forneceu uma base sólida para a avaliação objetiva dos movimentos do pé.

Embora a maior parte da população faça uso regular de calçados durante a realização de suas atividades rotineiras, os modelos descritos para a avaliação dos movimentos do pé usualmente avaliam os indivíduos deambulando sem o uso de calçados, o que pode influenciar significativamente os parâmetros de avaliação ou mesmo os parâmetros cinemáticos da marcha humana. Tais avaliações podem, portanto, determinar resultados que não refletem, da melhor maneira, a movimentação usual dos segmentos do pé, sendo que a avaliação desses segmentos com o indivíduo calçado poderá fornecer informações mais fidedignas acerca do padrão cinemático do pé no dia a dia $(12,13)$.

Para tanto, um modelo que seja capaz de avaliar os diferentes segmentos do pé durante a marcha com os indivíduos calçados deve ser desenvolvido e testado para garantir a reprodutibilidade de suas medidas. Dessa forma, o objetivo do presente estudo foi desenvolver um modelo biomecânico composto por perna, retropé e antepé para avaliação do deslocamento angular dos segmentos do pé durante a marcha com indivíduos calçados e avaliar a confiabilidade teste-reteste das medidas obtidas.

\section{Materiais e métodos}

\section{Delineamento do estudo}

Foi realizado um estudo do tipo metodológico no Laboratório de Análise de Movimento da Escola de Educação Física, Fisioterapia e Terapia Ocupacional da Universidade Federal de Minas Gerais (UFMG).

\section{Amostra}

A amostra do tipo conveniência foi composta por voluntários jovens, recrutados na comunidade de acordo com os seguintes critérios de inclusão: (i) ter idade entre 18 e 30 anos; (ii) utilizar no dia a dia tênis com tamanhos entre 37 e 39 (medida brasileira); (iii) não ter sofrido lesões ou ter sido submetido a qualquer tipo de cirurgia de membros inferiores; (iv) não apresentar deficit visuais significativos não corrigidos por lentes corretivas; (v) não possuir alterações musculares ou neurológicas que interfiram no desempenho da marcha; (vi) aceitar participar da pesquisa e assinar o Termo de Consentimento Livre e Esclarecido (TCLE), previamente aprovado pelo Comitê de Ética em Pesquisa da UFMG: ETIC 0047.0.203.000-10. Os indivíduos foram excluídos quando houve relato de desconforto durante qualquer procedimento da coleta de dados.

\section{Análise Cinemática}

As variáveis cinemáticas de desfecho do estudo foram valores máximo e mínimo e amplitude de movimento (ADM) total nos planos frontal e transverso de antepé em relação ao retropé e nos planos sagital, frontal e transverso do retropé em relação à perna durante a fase de apoio da marcha. Cada indivíduo participou de duas coletas, com intervalo de sete dias. Esse procedimento foi necessário para permitir a verificação do grau de associação entre os dados a partir do cálculo dos coeficientes de confiabilidade teste-reteste.

O deslocamento angular de antepé em relação ao retropé e retropé em relação à perna foi obtido com o uso do sistema de análise de movimento Qualisys - Pro Reflex MCU (QUALISYS MEDICAL AB, 41112 Gothenburg, Suécia). A coleta envolveu o uso de oito câmeras com iluminação produzida por um grupo de diodos emissores de luz infravermelha, localizados em volta da lente de cada uma das câmeras. O Sistema Qualisys permite a reconstrução em três dimensões de marcadores passivos refletores localizados em proeminências ósseas especificas. Os marcadores passivos refletores captam e refletem a luz infravermelha, que é então captada pela lente das câmeras. Foi utilizada a frequência de coleta de $120 \mathrm{~Hz}$. Sincronizado ao sistema Qualisys, uma plataforma de força AMTI ${ }^{\circledR}$ (Advanced Mechanical Tecnology, modelo OR6-6, Watertown, MA, USA) embutida na passarela foi utilizada para captar os dados da força de reação de solo necessários para a delimitação da fase de apoio da marcha. A calibração foi realizada de acordo com o manual do fabricante e foram permitidos erros de desvio padrão menores que $10 \mathrm{~mm}$ (14).

Para a construção do modelo biomecânico composto por antepé, retropé e perna, marcadores reflexivos $(9 \mathrm{~mm})$ foram afixados nos epicôndilos lateral e medial do fêmur, maléolo lateral e medial, sustentáculo do tálus, tróclea fibular, base 
e cabeça do primeiro e quinto metatarsos, estando inicialmente o indivíduo descalço. Para possibilitar o rastreamento dos segmentos durante a marcha, clusters foram fixados com faixa elástica em regiões específicas: (i) um cluster semirrígido composto por três marcadores refletores foi posicionado no terço médio da perna, (ii) um cluster rígido com três marcadores foi posicionado sobre o calcâneo, próximo à inserção do tendão calcâneo e, no segmento antepé, (iii) um cluster rígido com três marcadores foi posicionado sobre a região correspondente às diáfises dos segundo e terceiro metatarsos. Uma coleta estática foi conduzida para o registro da posição inicial e orientação dos marcadores anatômicos e de rastreamento. A coleta estática foi realizada com o indivíduo descalço em posição ortostática, o que possibilitou a utilização dos marcadores de definição e de rastreamento dos segmentos do pé e da perna durante a coleta (Figura 1). A palpação das proeminências ósseas e fixação dos marcadores

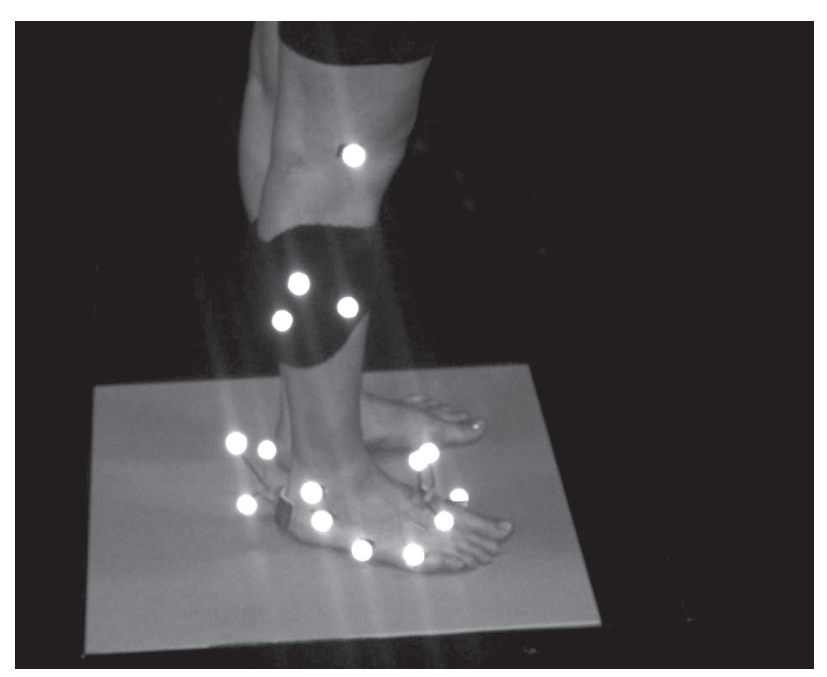

Figura 1 - Coleta estática com o indivíduo descalço Fonte: Dados da pesquisa.

\section{Características dos tênis utilizados}

Um par de tênis, tamanho 39 (medida brasileira), desenvolvido especificamente para a prática de corrida e caminhada, foi adquirido para a realização do estudo. 0 par de tênis teve suas linguetas retiradas para possibilitar o uso do cluster de antepé e, em sua parte posterior, foi realizada uma abertura posteriormente fixada com velcro na parte superior do talão do tênis com o objetivo de possibilitar o uso foram realizadas sempre pelo mesmo examinador, o qual possuía experiência com o processo de coleta de dados. Todos os ângulos reportados foram relativos a essa posição estática de referência.

Após a coleta estática, os marcadores de epicôndilos lateral e medial do fêmur, maléolo lateral e medial, sustentáculo do tálus, tróclea fibular, base e cabeça do $1^{\circ}$ e $5^{\circ}$ metatarsos foram retirados para que pudessem ser realizadas as coletas dinâmicas. Um avaliador previamente treinado foi responsável por retirar os marcadores iniciais e calçar o tênis nos participantes sem que houvesse movimento dos clusters de rastreamento de perna, retropé e antepé. Após a colocação dos tênis, foi permitido um período de cinco minutos para que o indivíduo se adaptasse ao tênis, à passarela e aos marcadores; após esse período, iniciaram-se as coletas dinâmicas nas quais um mínimo de cinco passadas em velocidade autosselecionada foi coletado em uma passarela de dez metros de comprimento (Figura 2).

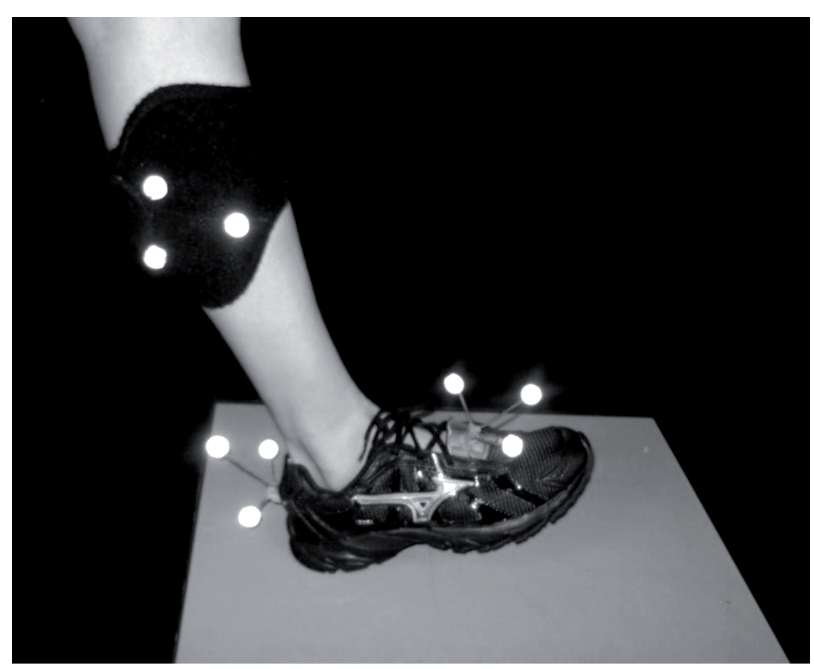

Figura 2 - Coleta dinâmica com o indivíduo calçado Fonte: Dados da pesquisa.

do cluster de retropé posicionado sobre o calcâneo. Essa fixação com velcro era aberta no momento de calçar o tênis no participante e fechada após a colocação do calçado. Após a fixação, a abertura apresentou dimensões de 2,8 $\mathrm{cm}$ por 2,0 cm. Essas adaptações possibilitaram a manutenção dos clusters de rastreamento entre a coleta estática e dinâmica sem modificação da posição inicial dos mesmos com mínima alteração da firmeza proporcionada pelo tênis durante a marcha. 
Redução dos dados

Os dados cinemáticos foram capturados pelo software de aquisição Qualisys Track Manager 1.9.2. Nessa etapa, realizou-se a interpolação quando a trajetória dos marcadores foi perdida por no máximo 10 quadros. Foram excluídas as passadas que apresentaram algum artefato, como má qualidade do sinal. Todos os dados coletados foram processados por um segundo examinador, sendo este cegado em relação aos indivíduos e ao dia da coleta com uso de códigos associados a cada voluntário.

Em seguida, os dados foram exportados para o software Visual3D C-Motion, Inc para seu processamento, utilizando as rotações padronizadas de Euler (X-Y-Z). Foram realizadas a construção do modelo biomecânico dos segmentos corporais e a aplicação desse modelo nos arquivos dinâmicos. Os dados da plataforma de força foram processados e utilizados para a normalização da fase de apoio da marcha, de 0 a 100\% (contato do pé à retirada do mesmo pé do solo).

Os ângulos articulares foram calculados usando-se a sequência de Cardan e definidos a partir da orientação do sistema de coordenadas de um segmento corporal em relação ao sistema de coordenadas do segmento de referência. A perna foi utilizada como segmento referência para o cálculo do ângulo do retropé, e este foi utilizado como segmento referência para o cálculo do ângulo do antepé. Em seguida, foi aplicado um filtro passa-baixa Butterworth de quarta ordem com frequência de corte estabelecida em $6 \mathrm{~Hz}$ (15). Foram gerados os gráficos da média dos deslocamentos angulares nos três planos do retropé e nos planos frontal e transverso de antepé durante a fase de apoio da marcha. Para a geração dos gráficos, foi utilizada a convenção de deslocamentos angulares positivos para dorsiflexão de retropé e inversão e adução de retropé e antepé.

Os dados foram exportados para o programa Microsoft Excel, onde foram calculados os valores angulares máximos e mínimos e as ADM totais a partir da subtração do menor valor angular do maior valor angular durante a fase de apoio da marcha.

\section{Análise estatística}

Os dados antropométricos dos participantes foram descritos por medidas de tendência central e de dispersão com o objetivo de caracterização da amostra. As médias dos valores máximos, mínimos e de ADM das duas visitas e a variabilidade intra e intersujeito foram apresentadas. As medidas de concordância foram expressas em termos de coeficientes de correlação e a análise estatística dos dados foi feita utilizando-se o Coeficiente de Correlação Intraclasse (ICC). Foi calculada a confiabilidade teste-reteste para as variáveis de desfecho do estudo. A força ou magnitude da relação entre as medidas foi classificada como fraca (ICC $<0,4$ ), moderada $(0,4 \leq$ ICC $\leq 0,75)$ e forte (ICC $>0,75)(16)$. Na maior parte dos estudos, valores de confiabilidade maiores do que $40 \%$ são necessários para prover um método de medida útil clinicamente (17). Todos os cálculos foram realizados utilizando o programa estatístico SPSS para Windows versão 15.0, com nível de significância estabelecido de $\alpha=0,05$.

\section{Resultados}

Amostra e variáveis espaçotemporais

Participaram do estudo dez adultos jovens saudáveis (sete do sexo feminino e três do sexo masculino) com média de idade de 25 anos ( $D P=3,3$ ), média de massa e de altura de $63 \mathrm{~kg}(\mathrm{DP}=8,9)$ e $1,70 \mathrm{~m}$ (DP $=0,04)$, respectivamente. Baseado em Walter et al. (17), dez indivíduos são suficientes para a realização de um estudo de confiabilidade com um alfa de 0,05 e beta de 0,2 .

As velocidades de marcha apresentadas pelos indivíduos foram similares entre as duas condições experimentais durante ambas as coletas, permitindo comparação adequada dos resultados sem influência da velocidade. Durante a primeira visita, os indivíduos apresentaram velocidade média de $1,27 \mathrm{~m} / \mathrm{s}$ (DP = 0,13 ; IC 95\% [1,17-1,37]) e comprimento da passada de 1,38 m (DP = 0,11; IC 95\% [1,30-1,47]); durante a segunda visita, eles apresentaram 1,37 m/s (DP = 0,12; IC 95\% [1,28-1,47]) e 1,46 m (DP = 0,04; IC95\% [1,43-1,50]) de velocidade média e comprimento da passada, respectivamente.

\section{Confiabilidade}

Os valores angulares máximos e mínimos e as ADM totais médias das duas visitas e a variabilidade 
intrassujeito e intersujeito foram apresentados no Quadro 1. A variabilidade intrassujeito foi de 0,99 a 2,37 para ADM, entre 0,77 e 2,29 para valores máximos e entre 0,77 e 1,53 para valores mínimos. Além disso, pôde-se observar que as variáveis de desfecho do estudo apresentaram confiabilidade teste-reteste variando entre moderada e excelente, e que a mensuração da ADM total, valor máximo e mínimo do retropé no plano sagital apresentaram os maiores valores de ICC entre as variáveis de desfecho do estudo. Os valores de ICC para cada variável de desfecho do estudo foram apresentados no Quadro 2.

\section{Retropé-perna}

No contato inicial, o retropé apresentou-se em dorsiflexão no plano sagital, discreta inversão no plano frontal e abdução no plano transverso. Durante os $10 \%$ inicias da fase de apoio, o retropé moveu-se em flexão plantar, máxima eversão e abdução. Máxima flexão plantar e posterior dorsiflexão, inversão e abdução caracterizaram o período entre 10 e $20 \%$ da fase de apoio. Entre 20 e $60 \%$ da fase de apoio, ocorreram dorsiflexão, inversão e adução do retropé. Entre 60 e $80 \%$ da fase de apoio, o retropé moveu-se para máxima dorsiflexão e permaneceu sem alterações significativas nos planos frontal e transverso. Na fase final de apoio (80 a 100\%), ocorreram flexão plantar, máxima inversão e abdução (Gráfico 1).

\section{Antepé-retropé}

O antepé estava invertido no plano frontal e aduzido no plano transverso no contato inicial. Durante os 10\% inicias da fase de apoio, o antepé apresentou máxima inversão e máxima adução. Eversão e discreta abdução caracterizaram o período entre 10 e $20 \%$ da fase de apoio. Entre 20 e $60 \%$, ocorreram eversão e abdução. Entre 60 e 80\%, ocorreram discreta inversão e adução. Na fase final de apoio (80 a 100\%), ocorreram máxima eversão com posterior inversão e discreta abdução (Gráfico 2).

Quadro 1 - Média (em graus) de ADM, valores máximo e mínimo (DP) para as diferentes articulações nos diferentes planos e variabilidade intrassujeito (média de DP dos indivíduos nas duas visitas)

\begin{tabular}{|c|c|c|c|c|c|c|}
\hline & \multicolumn{2}{|c|}{ ADM } & \multicolumn{2}{|c|}{ Valor máximo } & \multicolumn{2}{|c|}{ Valor mínimo } \\
\hline & Média & Variabilidade & Média & Variabilidade & Média & Variabilidade \\
\hline \multicolumn{7}{|l|}{ Retropé } \\
\hline Sagital & $24,8(7,8)$ & 0,99 & $35,7(15,2)$ & 0,77 & $9,0(13,2)$ & 0,97 \\
\hline Frontal & $10,4(3,2)$ & 2,37 & $6,3(7,1)$ & 2,29 & $-4,5(6,9)$ & 1,53 \\
\hline Transverso & $13,7(3,8)$ & 1,14 & $-14,9(5,4)$ & 1,11 & $-28,5(7,2)$ & 0,91 \\
\hline \multicolumn{7}{|l|}{ Antepé } \\
\hline Frontal & $6,8(1,9)$ & 1,32 & $4,0(7,0)$ & 1,09 & $-2,8(7,5)$ & 1,04 \\
\hline Transverso & $4,1(1,7)$ & 1,24 & $9,5(7,4)$ & 1,34 & $5,5(6,9)$ & 0,77 \\
\hline
\end{tabular}

Legenda: $\mathrm{ADM}=$ amplitude de movimento; $\mathrm{DP}=$ desvio padrão.

Fonte: Dados da pesquisa.

Quadro 2 - Valores de coeficiente de correlação intraclasse para cada variável de desfecho do estudo

(Continua)

\begin{tabular}{lccc}
\hline Segmento/Variável & ADM & Valor máximo & Valor mínimo \\
\hline Retropé sagital & 0,98 & 0,93 & 0,92 \\
Retropé transverso & 0,82 & 0,73 & 0,90 \\
Retropé frontal & 0,79 & 0,82 & 0,63 \\
\hline
\end{tabular}


Quadro 2 - Valores de coeficiente de correlação intraclasse para cada variável de desfecho do estudo

(Conclusão)

\begin{tabular}{lccc}
\hline Segmento/Variável & ADM & Valor máximo & Valor mínimo \\
\hline Antepé frontal & 0,65 & 0,80 & 0,79 \\
Antepé transverso & 0,60 & 0,72 & 0,75 \\
\hline
\end{tabular}

Legenda: $A D M=$ amplitude de movimento.

Fonte: Dados da pesquisa.

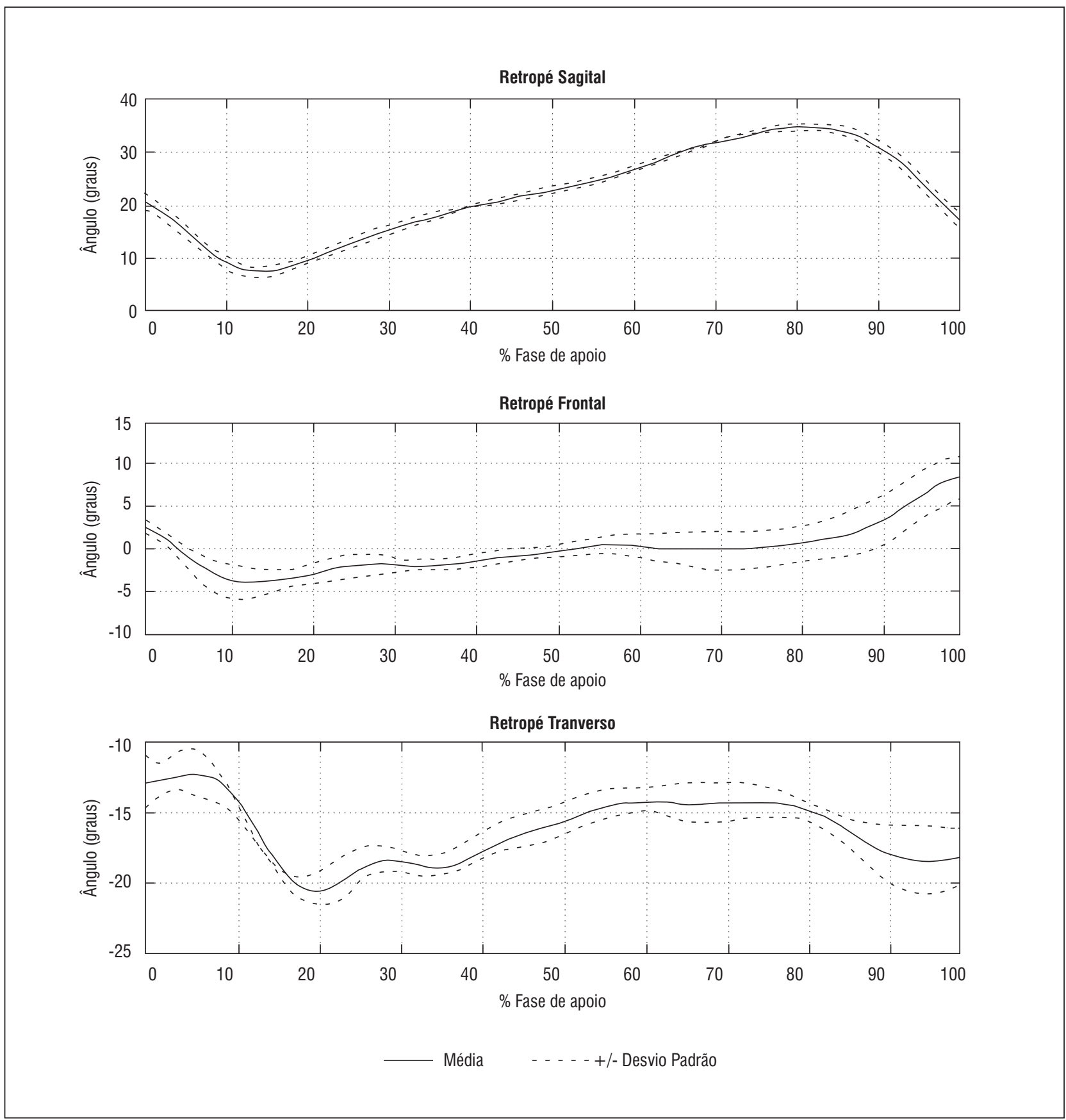

Gráfico 1 - Deslocamento angular do retropé durante a fase de apoio da marcha de um indivíduo representativo. Dorsiflexão $(+)$, flexão plantar (-); inversão (+), eversão (-); adução (+), abdução (-)

Fonte: Dados da pesquisa. 

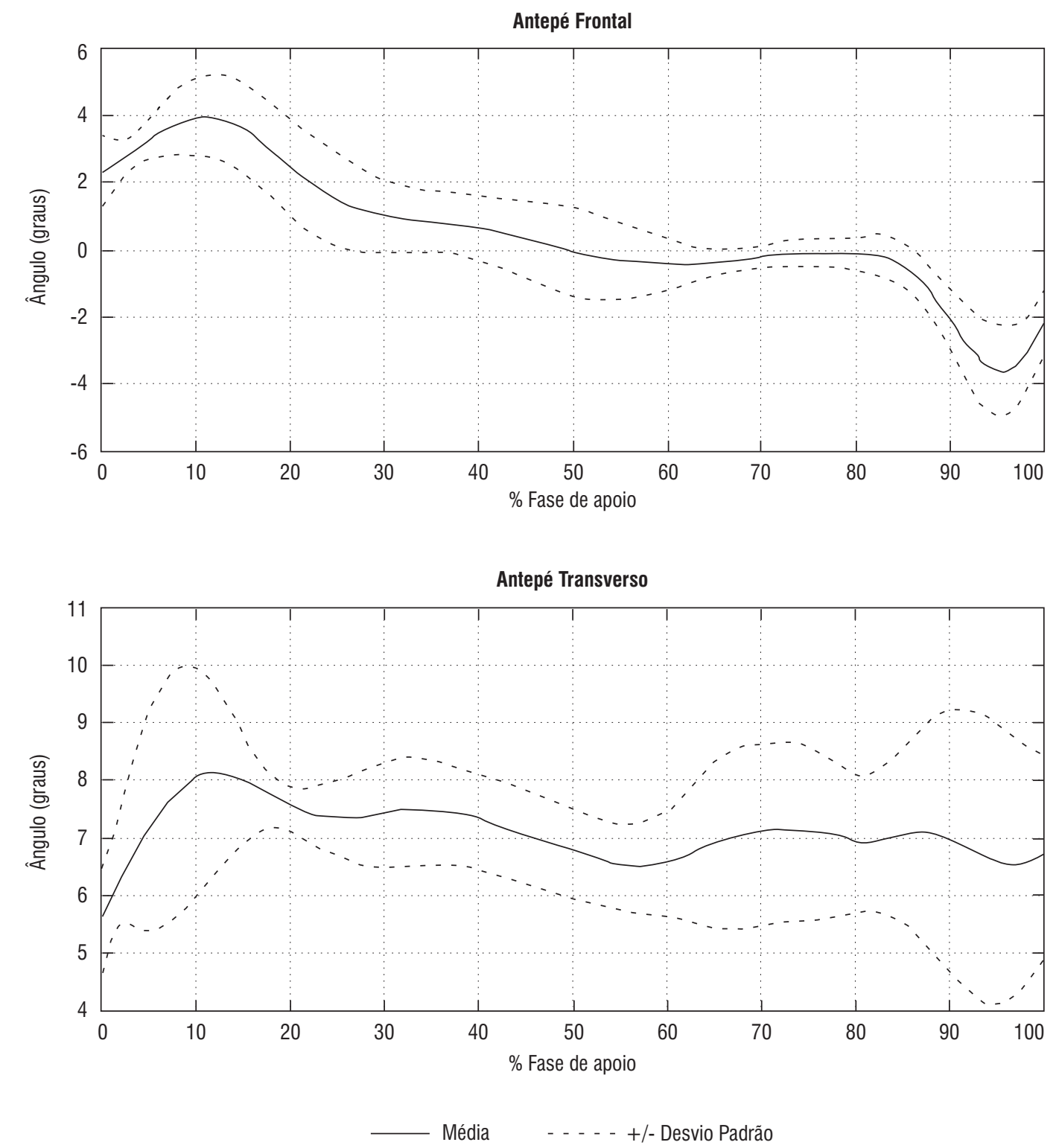

Gráfico 2 - Deslocamento angular do antepé durante a fase de apoio da marcha de um indivíduo representativo. Inversão (+), eversão (-); adução (+), abdução (-)

Fonte: Dados da pesquisa.

\section{Discussão}

O método apresentado no presente estudo possibilitou a avaliação do deslocamento angular em três dimensões dos segmentos do pé com o indivíduo calçado por meio de um sistema baseado em coordenadas de marcadores anatômicos. As variáveis de desfecho do presente estudo apresentaram confiabilidade teste-reteste de moderada a excelente, o que demonstra que o método apresentado é confiável para a avaliação do deslocamento angular dos segmentos do pé durante a fase de apoio da marcha com indivíduos calçados. Além disso, a variabilidade intrassujeito foi reproduzível, apesar do uso de marcadores de superfície e do movimento da pele. As diferenças entre sujeitos foram maiores, indicando que o modelo 
é sensível o suficiente para demonstrar a variabilidade entre sujeitos sem nenhuma deformidade aparente.

Embora seja importante a avaliação da confiabilidade dos métodos utilizados, uma apropriada análise de confiabilidade das medidas de cinemática dos segmentos do pé é encontrada em apenas alguns estudos (8). Segundo Bland e Altman (18), esse processo é recomendado especialmente nas ciências que avaliam o comportamento motor, uma vez que a maioria das avaliações envolve possíveis erros de mensuração relacionados à padronização dos procedimentos e características do examinador. A boa consistência observada entre as medidas em que se utilizou o presente modelo indica que os possíveis erros inerentes a seus procedimentos, como artefatos decorrentes da movimentação da pele, são sistemáticos, o que contribui para a adequada quantificação e interpretação das variáveis de desfecho do presente estudo.

Os clusters de marcadores rígidos utilizados possibilitaram melhor fixação sobre as estruturas ósseas definidas para o rastreamento dos segmentos do que marcadores fixados diretamente sobre a pele, o que possivelmente minimizou erros relacionados à movimentação da pele. Além disso, a orientação dos clusters permite a adequada visualização dos marcadores durante toda a fase de apoio da marcha; isso reduz a necessidade de utilização de filtros para interpolação das trajetórias desses marcadores após o término da coleta de dados. Apesar de os clusters terem permanecido fixos sobre as estruturas ósseas durante toda a coleta dos dados, nenhum sujeito avaliado relatou qualquer tipo de incômodo ou influência sobre a sua forma de andar em consequência do método utilizado.

A realização de aberturas no tênis é um dos métodos possíveis para medir o movimento dos segmentos do pé dentro do tênis (19). As dimensões das aberturas utilizadas no presente estudo foram similares às utilizadas por Stacoff et al. (19), que não encontraram diferenças nas medidas angulares obtidas em decorrência da realização das aberturas. Essas aberturas não podem ser muito pequenas a ponto de não permitirem a movimentação dos clusters de rastreamento nem muito grandes a ponto de reduzirem a rigidez do talão do tênis. As adaptações realizadas nos tênis são de fácil execução e não requerem maior investimento financeiro além daquele necessário para comprar os tênis. Além disso, durante toda a realização do estudo, nenhum indivíduo relatou insegurança, incômodo ou falta de firmeza para deambular com o tênis e com os clusters de rastreamento.
A forma de adaptação dos calçados no presente estudo fornece um método simples para mensuração da movimentação dos segmentos do pé com o indivíduo calçado, sem a necessidade de fabricação de calçados específicos para tal propósito. Esse método é importante, pois foi demonstrado na literatura que os movimentos do talão do tênis não são idênticos aos movimentos do calcâneo dentro do tênis (19). Dessa forma, com o objetivo de investigar possíveis relações entre o design do tênis e a cinemática dos segmentos do pé e a ocorrência de lesões, é recomendada a utilização de métodos que incluam aberturas realizadas no tênis para fixação dos marcadores diretamente na pele dos indivíduos (19).

Alguns modelos multissegmentados para o pé foram apresentados previamente na literatura $(8,11)$. No entanto, esses modelos tiveram como objetivo avaliar o deslocamento angular dos segmentos do pé com o indivíduo descalço. Apesar de possibilitar maior segmentação do pé, avaliar o deslocamento angular dos segmentos do pé com o indivíduo descalço pode não captar a influência do calçado utilizado pelo indivíduo; estudos prévios demonstraram que andar descalço ou calçado pode modificar o padrão de movimento dos segmentos do pé $(20,21,22)$, e que diferenças entre os tipos de calçados também podem alterar o padrão cinemático dos membros inferiores durante a marcha. Dessa forma, ter um método confiável para a avaliação do deslocamento angular dos segmentos do pé com o indivíduo calçado possibilitará maior entendimento da relação entre as características do calçado e a cinemática dos membros inferiores durante a marcha.

É esperado que métodos diferentes de segmentação do pé forneçam padrões cinemáticos distintos. Assim, torna-se difícil a comparação dos deslocamentos angulares obtidos com outros estudos em consequência de diferentes definições das referências anatômicas, da posição neutra do pé e das articulações analisadas. Além disso, alguns estudos reportaram os dados de somente um sujeito $(2,23)$, o que dificulta a extrapolação dos padrões cinemáticos obtidos. Ao contrário de alguns estudos anteriores (24), o protocolo de aquisição apresentado no presente estudo não é invasivo, não requer uma estrutura extra de referência $(7,11,25-31)$, posicionamento da articulação subtalar em neutro $(32,33)$ nem é dependente da informação fornecida por raios-X para definição da posição neutra de referência. Essas técnicas são complexas e demandam muito tempo para a realização 
da coleta de dados, além de, em alguns casos, expor o indivíduo à radiação. No entanto, possíveis alterações do alinhamento estrutural de retropé e antepé podem influenciar negativamente na definição dos eixos articulares baseados no protocolo de marcadores deste estudo. Além disso, os valores angulares máximos e mínimos encontrados podem não representar os valores exatos, pois as coletas dinâmicas foram analisadas tendo como base a coleta estática com os indivíduos descalços, o que, no entanto, não influencia no padrão de deslocamento angular e nos valores de ADM total reportados.

Um método simples e confiável para avaliar o deslocamento angular dos segmentos do pé durante a marcha com o indivíduo calçado foi apresentado no presente estudo. A qualidade do método desenvolvido e da análise de confiabilidade realizada detectou padrões de movimento dos segmentos do pé próximos aos descritos na literatura. 0 nível de confiabilidade encontrado foi considerado aceitável, fornecendo, assim, uma base sólida para a avaliação objetiva da cinemática dos segmentos do pé com indivíduos calçados, em ambientes laboratoriais, para fins de pesquisas científicas e avaliações clínicas da cinemática da marcha humana.

\section{Referências}

1. Gage JR, Deluca PA, Renshaw TS. Gait analysis: principle and applications with emphasis on its use in cerebral palsy. Instr Course Lect. 1996;45:491-507. PMid:8727765.

2. Kidder SM, Abuzzahab FS, Harris GF, Johson JE. A system for the analysis of foot and ankle kinematics during gait. IEEE Trans Rehabil Eng. 1996;4(1):25-32. doi:10.1109/86.486054.

3. Radin EL, Yang KH, Riegger C, Kish VL, O'Connor JJ. Relationship between lower limb dynamics and knee joint pain. J Orthop Res. 1991;9(3):398-405. doi:10.1002/jor.1100090312.

4. Kirkwood RN, Resende RA, Magalhães CMB, Gomes HA, Mingoti SA, Sampaio RF. Application of principal component analysis on gait kinematics in elderly women with knee osteoarthritis. Rev Bras Fisioter. 2011;15(1):52-8. doi:10.1590/S141335552011000100007.
5. Nigg BM, Cole GK, Nachbauer W. Effects of arch height of the foot on angular motion of the lower extremities in running. J Biomech. 1993;26(8):909-16. doi:10.1016/0021-9290(93)90053-H.

6. Bellchamber TL, Van Den Bogert AJ. Contributions of proximal and distal moments to axial tibial rotation during walking and running. J Biomech. 2000;33(11):1397403. doi:10.1016/S0021-9290(00)00113-5.

7. Donatelli R, Wooden M, Ekedahl SR, Wilkes JS, Cooper J, Bush AJ. Relationship between static and dynamic foot postures in professional baseball players. J Orthop Sports Phys Ther. 1999;29(6):31625. PMid:10370915.

8. Carson MC, Harrington ME, Thompson N, O'Connor JJ, Theologis TN. Kinematic analysis of a multi-segment foot model for research and clinical applications: a repeatability analysis. J Biomech. 2001;34(10):1299307. doi:10.1016/S0021-9290(01)00101-4.

9. Resende RA, Kirkwood RN, Figueiredo EM. Cinemática da marcha de adultos jovens: dados normativos iniciais. Ter Man. 2010;8(39):370-6.

10. Scott SH, Winter DA. Talocrural and talocalcaneal joint kinematics and kinetics during the stance phase of walking. J Biomech. 1991;24(8):743-52. doi:10.1016/0021-9290(91)90338-N.

11. Leardini A, Benedetti MG, Catani F, Simoncini L, Giannini S. An anatomically based protocol for the description of foot segment kinematics during gait. Clin Biomech (Bristol. Avon.). 1999;14(8):528-36. doi:10.1016/S0268-0033(99)00008-X.

12. Jenkyn TR, Anas K, Nichol A. Foot segment kinematics during normal walking using a multisegment model of the foot and ankle complex. J Biomech Eng. 2009;131(3):034504. doi:10.1115/1.2907750.

13. Oeffinger D, Brauch B, Cranfill S, Hisle C, Wynn C, Hicks R, et al. Comparison of gait with and without shoes in children. Gait Posture. 1999;9(2):95-100. doi:10.1016/S0966-6362(99)00005-3.

14. Gard SA, Miff SC, Kuo AD. Comparison of kinematic and kinetic methods for computing the vertical motion of the body center of mass during walking. Hum Mov Sci. 2004;22(6):597-610. doi:10.1016/j. humov.2003.11.002. 
15. Winter DA. Biomechanical motor patterns in normal walking. J Mot Behav. 1983;15(4):302-30. PMid:15151864.

16. Portney LG, Watkins MP. Foundations of clinical research: aplications to practice. 2nd. ed. New Jersey: Prentice-Hall Health; 2000. p. 61-77.

17. Walter SD, Eliasziw M, Donner A. Sample size and optimal designs for reliability studies. Stat Med. 1998;17:101-10. doi:10.1002/(SICI)1097-0258 (19980115)17:1<101::AID-SIM727>3.3.CO;2-5.

18. Bland JM, Altman DG. Measurement error and correlation coefficients. BMJ. 1996;313:41-2. doi:10.1136/ bmj.313.7048.41.

19. Stacoff A, Reinschmidt C, Stüssi E. The movement of the hell within a running shoe. Med Sci Sports Exerc. 1992;24(6):695-701. doi:10.1249/ 00005768-199206000-00012.

20. Butler RJ, Davis IS, Hamill J. Interaction of arch type and footwear on running mechanics. Am J Sports Med. 2006;34(12):1998-2005. doi:10.1177/ 0363546506290401.

21. Hamill J, Bates BT, Holt KG. Timing of lower extremity joint actions during treadmill running. Med Sci Sports Exerc. 1992;24(7):807-13. doi:10.1249/00005768-199207000-00011.

22. Morio C, Lake MJ, Gueguen N, Rao G, Baly L. The influence of footwear on foot motion during walking and running. J Biomech. 2009;42(13):2081-8. doi: 10.1016/j.jbiomech.2009.06.015.

23. Delozier G, Alexander I, Narayanaswamy R. A method for measurement of integrated foot kinematics. International Symposium on Three-Dimensional Analysis of Human Movement, Montreal, Canada. 1991:79-82.

24. Chen WP, Tang FT, Ju CW. Stress distribution of the foot during mid-stance to push-off in barefoot gait: a 3-D finite element analysis. Clin Biomech. 2001;16(7):61420. doi:10.1016/S0268-0033(01)00047-X.

25. Rattanaprasert U, Smith R, Sullivan M, Gilleard W. Three-dimensional kinematics of the forefoot, rearfoot, and leg without the function of tibialis posterior in comparison with normals during stance phase of walking. Clin Biomech. 1999;14(1):14-23. doi:10.1016/S0268-0033(98)00034-5.
26. Stebbins J, Harrington M, Thompson N, Zavatsky A, Theologis T. Repeatability of a model for measuring multi-segment foot kinematics in children. Gait Posture. 2006;23(4):401-10. doi:10.1016/j. gaitpost.2005.03.002.

27. Hunt AE, Smith RM, Torode M. Extrinsic muscle activity, foot motion and ankle joint moments during the stance phase of walking. Foot Ankle Int. 2001;22(1):31-41. PMid:11206820.

28. Kaufman K, Morrow D, Hansen D, Kitaoka HB. Technique for measurement of foot ankle kinematics. Gait and Clinical Movement Analysis Society - GCMAS. 2001; Sacramento, USA.

29. Moseley L, Smith R, Hunt A, Gant R. Threedimensional kinematics of the rearfoot during the stance phase of walking in normal young adult males. Clin Biomech. 1996;11(1):39-45. doi:10.1016/ 0268-0033(95)00036-4.

30. Simon J, Doederlein L, McIntosh AS, Metaxiotis D, Bock HG, Wolf SI. The Heidelberg foot measurement method: development, description and assessment. Gait Posture. 2006;23(4):411-24. doi:10.1016/j. gaitpost.2005.07.003.

31. Theologis TN, Harrington ME, Thompson N, Benson MK. Dynamic foot movement in children treated for congenital talipes equinovarus. J Bone Joint Surg Br. 2003;85(4):572-7. doi:10.1302/ 0301-620X.85B4.13696.

32. Kepple TM, Stanhope SJ, Lohmann KN, Roman NL. A videobased technique for measuring ankle-subtalar motion during stance. J Biomed Eng. 1990;12(4):273-80. doi:10.1016/0141-5425(90)90001-4.

33. Woodburn J, Turner DE, Helliwell PS, Barker S. A preliminary study determining the feasibility of electromagnetic tracking for kinematics at the ankle joint complex. Rheumatology (Oxford). 1999;38(12):12608. doi:10.1093/rheumatology/38.12.1260.

Recebido: $15 / 12 / 2011$ Received: 12/15/2011

Aprovado: 16/07/2012 Approved: 07/16/2012 